\title{
Chaos in Nonlinear Dynamo Models
}

\author{
J. KURTHS ${ }^{1}$, A. BRANDENBURG ${ }^{2}$, U. FEUDEL ${ }^{1}$, W. JANSEN ${ }^{1}$ \\ 1 Arbeitsgruppe Nichtlineare Dynamik der Max-Planck-Gesellschaft \\ an der Universität Potsdam, Am Neuen Palais, D-O-1571 Potsdam, Germany \\ ${ }^{2}$ NORDITA, Blegdamsvej 17, DK-2100 Copenhagen $\emptyset$, Denmark
}

\begin{abstract}
Two nonlinear dynamos have been analyzed by numerical means: 3D-simulation of the magneto-hydrodynamic equations and qualitative analysis of a simplified low-dimensional mean field model. It turns out that both are capable of deterministic chaos in a certain parametcr range. As the basic tool the calculation of Lyapunov exponents has been used.
\end{abstract}

\section{Introduction}

Self-organization has been regarded as one of the main features of evolutionary processes. The theory of dynamical systems is a suitable tool to describe such self-organizing processes because they are capable of creating new structures as parameters of the system change. If new structures spontaneously emerge at a critical parameter value, this kind of evolution is called a transition phenomenon. In particular, transitions from regular to complex (chaotic) behaviour, which are often reported in the literature (cf. Schuster, 1984), can only be explained through nonlinear interactions within a system far away from equilibrium. Using central quantities of the theory of nonlinear dynamics as fractal dimensions and Lyapunov exponents, we can distinguish different structures and the transitions between them in a unique manner (cf. Ruelle, 1989) (Section 2).

The solar activity is an interesting example of a self-organizing system. It is caused by the interaction between the motion of charged particles and magnetic fields. These processes of magnetohydrodynamics can be explained in the framework of dynamo theory (Krause \& Rädler, 1980). In Section 3 we study two nonlinear dynamo models to describe several aspects of creating structures.

\section{Characteristics of Nonlinear Systems}

Complex systems can often be described by a finite number of modes or generalized coordinates. It is instructive to represent the dynamics of such a system in an abstract phase space spanned by these modes. The number of variables $n$ defines the dimension of the phase space. A steady state of the system becomes a point in this space, whereas the evolution in time defines a curve, the trajectory. As time proceeds, trajectories of a deterministic dissipative system often reach an invariant regime, the attractor, and remain there forever unless changes of the parameters of the system occur. Simple attractors corresponding to well-ordered motions are either fixed points, indicating steady states, or limit cycles reflecting periodic motions. Aperiodic behaviour refers to more complex attractors, and can only arise from the nonlinear nature of the system.

Various quantities, such as (fractal) dimensions, Lyapunov exponents and Kolmogorov entropy, describe different aspects of the attractor structure. Methods taken from the theory of linear systems, such as power spectra and correlation 
functions, are often not sufficient to distinguish between nonlinear deterministic systems and stochastic ones (cf. Ruelle, 1989).

1. Dimensions refer to the geometric properties of an attractor. To determine a dimension, the structure of an attractor is examined under different levels of magnification. If the patterns observed at these different levels are essentially indistinguishable, this kind of scaling, called self-similarity, yields the capacity or box dimension $D_{B}$. Loosely speaking, the dimension indicates the number of independent variables which characterize the motion on the attractor. The dimension of complicated attractors can be a finite, often fractal, number, in which case we speak of a strange attractor. A stochastic process, on the other hand, has generally no finite dimension.

2. Lyapunov exponents are global dynamical invariants, based on a stability analysis of the system. The maximum Lyapunov exponent $\lambda_{1}$ describes the evolution of small perturbations. A positive $\lambda_{1}$ expresses an exponential divergence of initially nearby trajectories. A system with such a sensitive dependence on initial conditions is defined to be chaotic.

Both concepts together, dimensions and Lyapunov exponents, provide an efficient tool for a quantitative diagnosis of dynamical systems. In the case of a fixed point all $\lambda_{i}$ are less than zero and $D_{B}$ vanishes. If the dynamics on the attractor can be decomposed into $k$ incommensurate periods, the $k$ largest $\lambda_{i}$ are equal to zero and $D_{B}=k$. Chaotic systems $\left(\lambda_{1}>0\right)$ are often characterized by a fractal number $D_{B}$.

\section{Dynamo Models for Solar Activity}

Processes inside, on the surface of, and nearby our sun are examples of solar activity caused by the interaction between the motion of charged particles and magnetic fields which can be explained in terms of dynamo theory.

In the following we discuss two ways of studying the dynamics of the magnetic fields and the fluid motions. Firstly, equations for the mean magnetic field are studied to describe the large-scale variations of the solar activity. Secondly, the induction equation and the Navier-Stokes equation are solved and local effects in the dynamics within the convection zone are illustrated.

It is known that the equations of mean-field dynamo theory can have periodic solutions corresponding to regular dynamo waves, which provide the basis of an explanation of a periodic solar cycle. However, complicated amplitude- and frequency modulation is observed for solar activity which calls for a more general theory in which nonlinear and/or stochastic effects have to be included.

\subsection{A Nonlinear Mean-field Model}

Based on an approach presented by Zeldovich et al. (1983) Malinetzky et al. (1986) proposed a rather simplified nonlinear dynamo model obtained by truncation of the partial differential equations for the mean magnetic field. As the crucial point an additional effect has been included, namely the feedback of the magnetic field upon the helicity. The corresponding equation that expresses temporal changes of 
the helicity is highly nonlinear. Furthermore, the equations for the magnetic field become nonlinear, since the helicity is no longer a constant but varies with time. To make this model numerically tractable the equations are truncated by using a lowest-order mode ansatz. This yields a 7-dimensional system of autonomous differential equations for the coefficients of the first modes:

$$
\begin{aligned}
& \dot{a}_{1}=-\sigma a_{1}+\left(\alpha+c_{0}\right) b_{1}+\frac{1}{2}\left(c_{1} b_{1}+c_{2} b_{2}\right) \\
& \dot{a}_{2}=-\sigma a_{2}+\left(\alpha+c_{0}\right) b_{2}-\frac{1}{2}\left(c_{1} b_{1}-c_{2} b_{2}\right) \\
& \dot{b}_{1}=-b_{1}-D a_{2} \\
& \dot{b}_{2}=-b_{2}+D a_{1} \\
& \dot{c}_{0}=-\nu_{0} c_{0}+p\left(a_{1} b_{1}+a_{2} b_{2}\right)-q\left[\left(\alpha+c_{0}\right)\left(b_{1}^{2}+b_{2}^{2}\right)+\frac{1}{2} c_{1}\left(b_{1}^{2}-b_{2}^{2}\right)+c_{2} b_{1} b_{2}\right] \\
& \dot{c}_{1}=-\nu c_{1}+p\left(a_{1} b_{1}-a_{2} b_{2}\right)-q\left[\left(\alpha+c_{0}\right)\left(b_{1}^{2}-b_{2}^{2}\right)+c_{1}\left(b_{1}^{2}+b_{2}^{2}\right)\right] \\
& \dot{c}_{2}=-\nu c_{2}+p\left(a_{1} b_{2}+a_{2} b_{1}\right)-q\left[\left(\alpha+c_{0}\right) 2 b_{1} b_{2}+c_{2}\left(b_{1}^{2}+b_{2}^{2}\right)\right]
\end{aligned}
$$

The qualitative behaviour of this model has been analyzed by means of numerical methods implemented in the software system CANDYS/QA (Feudel \& Jansen, 1992). This software package is an efficient tool for studying invariant sets of nonlinear systems, such as steady-states, periodic, and quasi-periodic motions, their stability, and bifurcation phenomena.

For this model the dynamo number $D$ is the crucial bifurcation parameter. It is associated with all processes mentioned which play an important role in the solar magnetic cycle. Here, $D$ is proportional to differential rotation and mean helicity and is inversely proportional to the square of the coefficients of turbulent diffusivity. Additionally, the values at which qualitative transitions occur mainly depend on the parameter $q$ quantifying the influence of the higher-order terms.

As shown in the $D, q$-bifurcation diagram (Fig. 1) the qualitative behaviour of this rather simple model exhibits rich dynamics. Several different structures, such as steady-state, periodic, and quasi-periodic motions, are found to be stable in certain parameter regimes. The transitions from one structure to another correspond to bifurcations of different types, such as Hopf- and torus bifurcations. With increasing dynamo number we observe a more complicated nature of the solutions ending up in chaos (see Feudel et al., 1992). Lyapunov exponents $\lambda_{i}$ are used as the basic tool to distinguish quasi-periodic and chaotic structures.

The route to chaos which has been found to be typical for this system is characterized by the following transitions. Beyond the torus bifurcation a quasi-periodic motion on $T^{2}$ with two incommensurate frequencies arises which corresponds to two vanishing Lyapunov exponents $\left(\lambda_{1}=\lambda_{2}=0\right)$. With increasing dynamo number $D$ we obtain a transition $T^{2} \rightarrow T^{3}$ referring to a stable motion on $T^{3}$ with three incommensurate frequencies $\left(\lambda_{1}=\lambda_{2}=\lambda_{3}=0\right)$. For higher dynamo numbers the system returns again to a motion on $T^{2}$ due to a frequency locking (which occurs when the ratio of two frequencies becomes a rational number). This is followed by a torus doubling cascade which ends up in a chaotic motion $\left(\lambda_{1}>0\right)$. As Fig. 1 shows, we observe in all parameter regions the same route to chaos via quasi-periodicity, only the torus bifurcation and the onset of chaos are shifted towards lower values of the dynamo number $D$ with increasing influence of the higher order terms (i. e. 


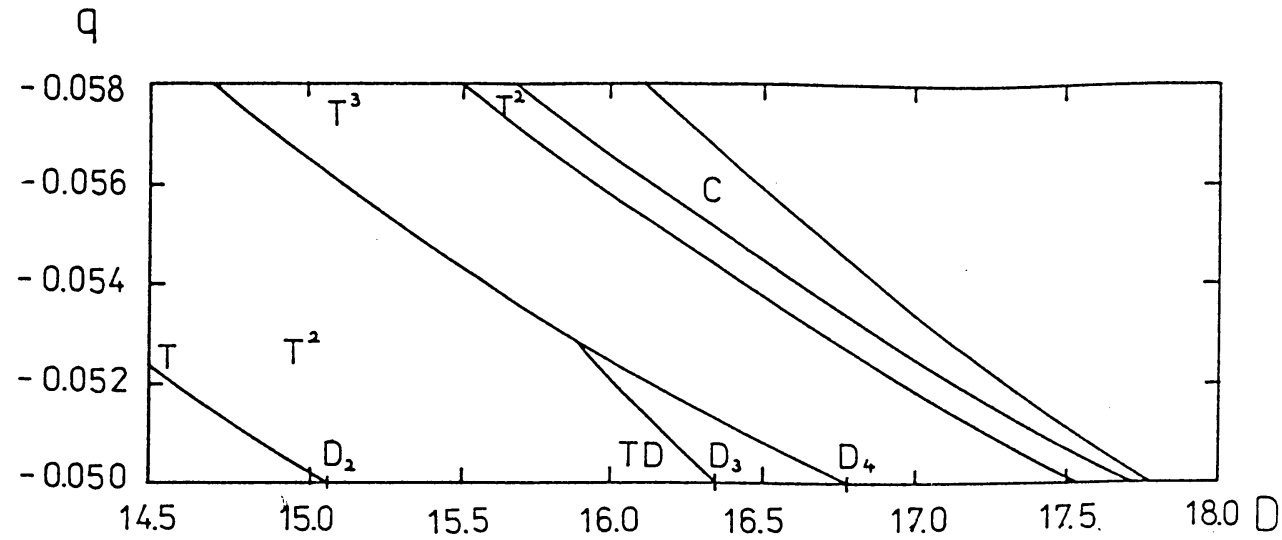

Fig. 1. Bifurcation diagram for the truncated nonlinear dynamo model dependent on the parameters $D$ and $q$.

$\mathrm{T}$ - torus bifurcation, TD - torus doubling, $\mathrm{T}^{2}$ - stable 2-torus, $\mathrm{T}^{3}$ - stable 3 -torus , $\mathrm{C}$ chaos $\left(\alpha=1.0, \sigma=1.0, p=-1.0, \nu=0.5, \nu_{0}=1.0\right)$ (adapted from Feudel et al., 1993).

increasing $q$ ).

A similar route to chaos has been reported by Jones et al. (1985) who studied a nonlinear dynamo model based on the feedback of the differential rotation upon the magnetic field.

\subsection{Simulation of Hydromagnetic Convection}

As is well-known from other areas, any simplification of the dynamo equations, such as mean-field theory, may suppress the essential nature of the process under consideration. An important progress in understanding hydromagnetic convection has come from the use of modern supercomputers, since they enable the treatment of the original three-dimensional (3D) hydromagnetic equations.

Indeed, it has been demonstrated by $3 \mathrm{D}$-simulations that spontaneous onset and maintenance of dynamo action can result from turbulent motions of a conducting fluid (Meneguzzi et al., 1989, Brandenburg et al., 1991, Nordlund et al., 1992). During the saturation phase there is a complicated balance of transfers between thermal, kinetic, and magnetic energies. This process is a typical example of selforganization.

Previously, we have shown that dynamo action occurs in a chaotic regime (Kurths and Brandenburg, 1991). To specify this kind of hydromagnetic convection we have calculated instantaneous divergence exponents $\lambda^{(i n s t)}(t)$. Starting from a small initial distance $d\left(t_{0}\right)$ of two trajectories at time $t_{0}$, which becomes the distance $d\left(t_{0}+t\right)$ after an evolution time $t$, we get the dilatation rate $\epsilon(t)=d\left(t_{0}+t\right) / d\left(t_{0}\right)$. It can always be written as $\epsilon(t)=e^{\lambda^{(i n * t)}(t) \cdot t}$. For deterministic systems these exponents converge to $\lambda_{1}$ as $t$ tends to infinity. In order to investigate spatial nonuni- 


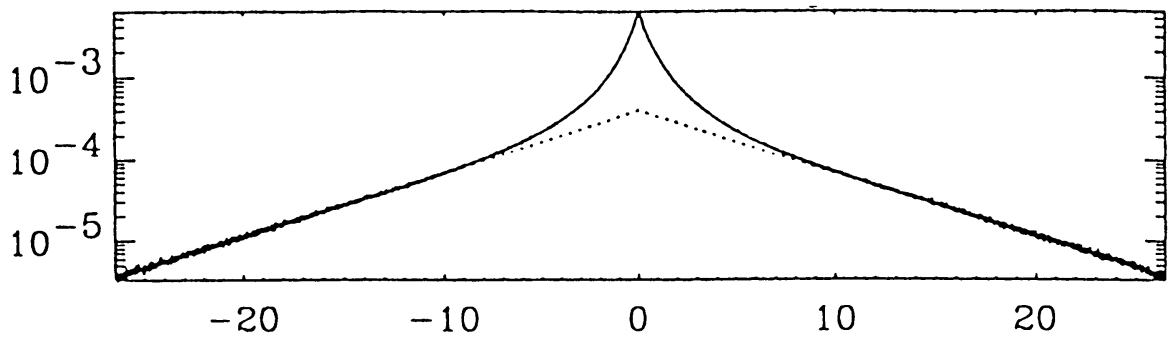

Fig. 2. Histogram of instantaneous and local divergence exponents $\lambda(\mathrm{x}, t)$ obtained from the 3 D-simulation (adapted from Kurths \& Brandenburg, 1991).

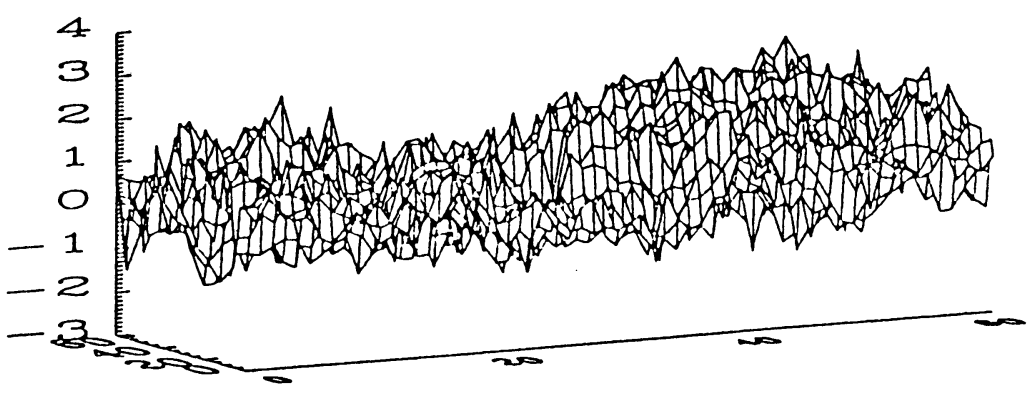

Fig. 3. Local divergence exponents $\lambda(\mathbf{x})$ obtained from the 3 D-simulation (adapted from Kurths \& Brandenburg, 1991).

formity we analogously define local and instantaneous exponents $\lambda(\mathbf{x}, t)$. The histogram of $\lambda(\mathbf{x}, t)$ obtained from the 3D-simulation is extremely broad, indicating an inhomogeneous nature of this process (Fig. 2). We observe a marked spatial nonuniformity of local exponents $\lambda(\mathbf{x})$ (Fig. 3). Unstable regions $(\lambda(\mathbf{x})>0)$ are often close to stable ones resulting in a pattern of $\lambda(\mathbf{x})$ that is interwoven in a complicated manner.

Video animations of this simulation show that the magnetic field is highly intermittent and organized into thin elongated flux tubes, undergoing repeated stretching and folding. This intermittency is expressed in a relatively high value of the kurtosis of the distribution of $\mathbf{B}(\mathbf{x}, t)$. Surprisingly, the kurtosis of the distribution of $\lambda(\mathbf{x}, t)$ is also very high and of similar magnitude.

\section{Conclusions}

We have analyzed qualitative properties of two nonlinear dynamo models by means of nonlinear dynamics. It comes out that both models are capable of deterministic 


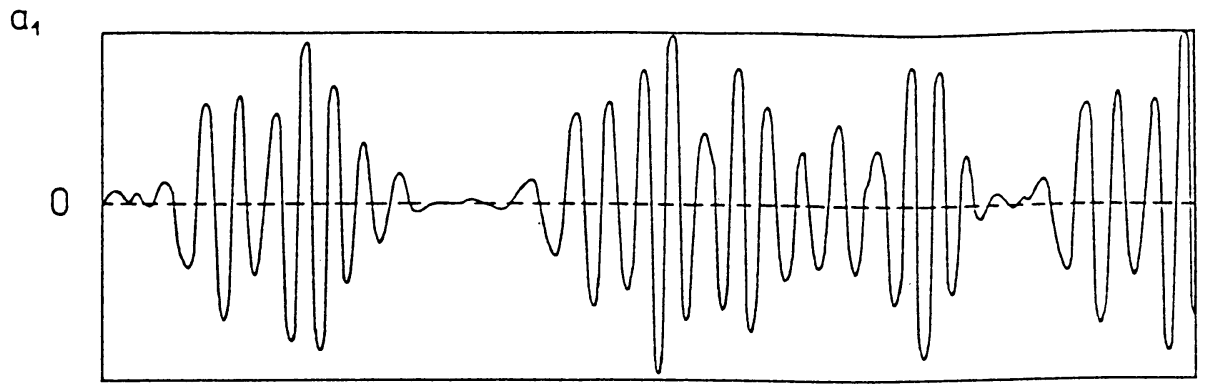

TIME

Fig. 4. Component $a_{1}$ depending on time in the chaotic regime in arbitrary units.

chaos in a certain parameter range.

We have got the following main results.

1. The 7-dimensional ordinary differential equation system obtained from a firstorder mode truncation of a nonlinear dynamo model for the mean magnetic field exhibits different kinds of qualitative behaviour. Beyond a Hopf bifurcation point, there occurs periodic, quasi-periodic, and chaotic behaviour. We find that the route to chaos via the transitions $T^{2} \rightarrow T^{3} \rightarrow T^{2} \rightarrow$ chaos is typical for this system. The trajectories calculated from the chaotic state display aperiodic cycles characterized by complicated amplitude and frequency modulation as well as epoches with very low amplitudes (Fig. 4). Such intervals of extremely low solar activity may be related to the grand minima reported by Eddy (1976).

2. For the second model basing on simulations of the 3D hydromagnetic equations, we have shown that dynamo action occurs in a chaotic regime. The analysis of instantaneous and local divergence exponents helps us to understand the relevant dynamics, in particular to describe the intermittent nature of the magnetic field.

It is, however, important to note that there is still a large gap to relate both kinds of models to each other. There are two reasons for this gap.

- It is very complicated or even impossible to perform a complete bifurcation analysis for high-dimensional systems. Due to the hugh computational effort needed, only some parts of the bifurcation phenomena can be investigated in this case.

- All results depend on the reduction scheme from partial differential equations to ordinary ones. Several techniques should be used to improve the approximation of the solutions of the partial differential equations.

From the viewpoint of nonlinear dynamics, a more complete analysis of the original dynamo equations will be possible if improved approaches to these two points become available. 


\section{References}

Brandenburg, A., Jennings, R. L., Nordlund, A. and Stein, R.F.: 1991, in T. Riste and D. Sherrington, ed(s)., Spontaneous formation of space-time structures and criticality, Nato ASI Series, Dordrecht, 371

Eddy, J.A.: 1976, Science 286, 1198

Feudel, U. and Jansen, W.: 1992, Int. J. Bifurcation \& Chaos 2,

Feudel, U., Jansen, W., and Kurths, J.: 1993, Int. J. Bifurcation \& Chaos 3, in press

Jones, C. A. and Weiss, N. O. and Cattaneo, F.: 1985, Physica 14D, 161

Krause, F. and Rädler, K.-H.: 1980, Mean-Field Magnetohydrodynamics and Dynamo Theory, Akademie-Verlag, Berlin

Kurths, J. and Brandenburg A.: 1991, Phys. Rev. A44, R3427

Malinetzky, G.G., Ruzmaikin, A.A. and Samarsky, A.A.: 1986, A Model for Long Variations of the Solar Activity, Preprint

Meneguzzi, M. and Pouquet, A.: 1989, J. Fluid Mech. 205, 297

Nordlund, A., Brandenburg, A., Jennings, R. L., Rieutord, M., Ruokolainen, J., Stein, R. F. and Tuominen, I.: 1992, Astrophys. J. 392, 647

Ruelle, D.: 1989, Chaotic Evolution and Strange Attractors, Cambridge University Press

Schuster, H.G.: 1984, Deterministic Chaos, VCH, Weinheim

Zeldovich, Ya. B., Ruzmaikin, A. A. and Sokoloff, D. D.: 1983, Magnetic Fields in Astrophysics, Gordon \& Breach, New York 